\title{
Effect of fibre laser marking on surface properties and corrosion resistance of a $\mathrm{Fe}-\mathrm{Ni}-\mathrm{Cr}$ alloy
}

\author{
Antonello Astarita ${ }^{1, \text { a) }}$, Chiara Mandolfino ${ }^{2, \text { b) }}$, Enrico Lertora ${ }^{2, c)}$, Carla \\ Gambaro $^{2, \mathrm{~d})}$, Antonino Squillace ${ }^{1, \mathrm{e}}$, Fabio Scherillo, \\ ${ }^{1}$ University of Naples Federico II, Department of Chemical, Materials and Production Engineering, P.le Tecchio 80, \\ Naples, Italy \\ ${ }^{2}$ University of Genoa Genova, Polytechnic School, Department of Mechanical Engineering, Via Opera Pia 15, \\ Genoa, Italy. \\ a) Corresponding author: antonello.astarita@unina.it \\ b) chiara.mandolfino@unige.it \\ c)e.lertora@unige.it \\ d) gambaro@dime.unige.it \\ e)squillac@unina.it \\ f)fabio.scherillo@unina.it
}

\begin{abstract}
Fiber laser techniques are increasing their use in many applications, including modification of material surface properties. In particular they are often used for materials' marking as a non-contact processing. In spite of this, the impact of the laser beam on the surface causes metallurgical and morphological changes. The developments during the laser-material interaction can also affect other surface properties, especially corrosion properties which are crucial in the case of Iron-Nickel alloys. Effect of laser marking on a Fe-Cr-Ni alloy using a Tm-fibre laser (IPG Photonics TRL1904; maximum power: 50W, wavelength: $1904 \mathrm{~nm}$ ), is described in this paper. In order to evaluate the effect of the laser on corrosion properties a specific ageing test in salt spray has been performed. Moreover, superficial morphology analyses have been performed on samples before and after corrosion tests. Possibilities and limitations of laser marking on these alloys have been discussed, in particular from the point of view of the marked surface corrosion resistance preservation.
\end{abstract}

\section{INTRODUCTION}

$\mathrm{Fe}-\mathrm{Ni}-\mathrm{Cr}$ alloys are extensively used in industry due to their high strength at elevated temperatures and their excellent corrosion resistance properties. Subsequently, they find application in heat-treating and chemical plants [1]. Despite several advantages, in general, these kinds of alloys are considered difficult to process when melting phenomena are involved, especially those exhibiting precipitation hardening reactions [2]. Micro cracks have been observed to occur in the melted and heat affected regions [3]. They can generally be attributed to liquidus cracking and subsolidus ductility [4].

On the other hand, lasers are extensively used in surface treatment industry. This is due to the high concentration of energy which generates precision in operation and a controlled heating [5].

In particular laser marking is a widely used method to obtain permanent marks in order to preserve traceability and identification data [6]. In several applications, such as chemical plant industry, in order to guarantee the identification and the traceability of the products, a long-lasting marking process, which is not detrimental to the properties of the material, is essential. Laser marking offers several advantages over the traditional processes, such as: high precision, high repeatability, non-contact operation, high speed and high automation [7]. 
In order to ensure the mark permanent visibility also in the component service, it is essential to control properly the quality and the geometry of the mark, in order to preserve the mark from eventual aggressive environments (i.e. in presence of oxidation, corrosion and wear phenomena) in which the material would be used. The mark qualities, especially mark clarity, are very important in the marking process. Therefore, optimizing the process parameters to achieve productivity on one hard and the required surface quality on the other, is an important goal.

The aim of this paper is to study the laser marking of a Fe-Ni-Cr alloy, through a $50 \mathrm{~W}$ Tm-doped fibre laser. Several sets of process parameters were investigated to study the feasibility of the process and a salt spray corrosion test was performed to explore the influence of the laser marking on the surface properties of the material. A characterization campaign, aiming to investigate the mark geometry and to study the influence of the process parameters on the above mentioned geometry, was carried out. In particular detailed observations, performed through a confocal microscope, were carried out on marks obtained under different processing conditions.

\section{MATERIAL AND METHODS}

The material used in this experiment was a Fe-Ni-Cr alloy with the chemical composition listed in Table 1. The dimensions of the plates used were $100 \mathrm{~mm} \times 100 \mathrm{~mm} \times 3 \mathrm{~mm}$. Three plates were laser marked using an IPG Photonics TRL1904 laser source. The fibre laser maximum power is $50 \mathrm{~W}$ and it was used in continuous mode for all of the tests, the wavelength is $1904 \mathrm{~nm}$ and it was installed on a robot arm (Panasonic TA1600). Air was used as a shielding gas in order to protect the optic system.

\begin{tabular}{|c|c|}
\hline Element & Percentage (\%) \\
\hline $\mathrm{C}$ & 0.014 \\
\hline $\mathrm{Mn}$ & 0.15 \\
\hline $\mathrm{Si}$ & 0.48 \\
\hline $\mathrm{P}$ & 0.020 \\
\hline $\mathrm{S}$ & 0.000 \\
\hline $\mathrm{Cr}$ & 20.49 \\
\hline $\mathrm{Ni}$ & 30.33 \\
\hline $\mathrm{Cu}$ & 0.030 \\
\hline $\mathrm{Ti}$ & 0.27 \\
\hline $\mathrm{Al}$ & 0.34 \\
\hline
\end{tabular}

On each sample ten simple lines and three rectangular areas of $10 \mathrm{~mm} \times 20 \mathrm{~mm}$ were produced, keeping the laser power constant at $40 \mathrm{~W}$ and varying the travel speed of the robot arm, following the scheme reported in Fig.1A.

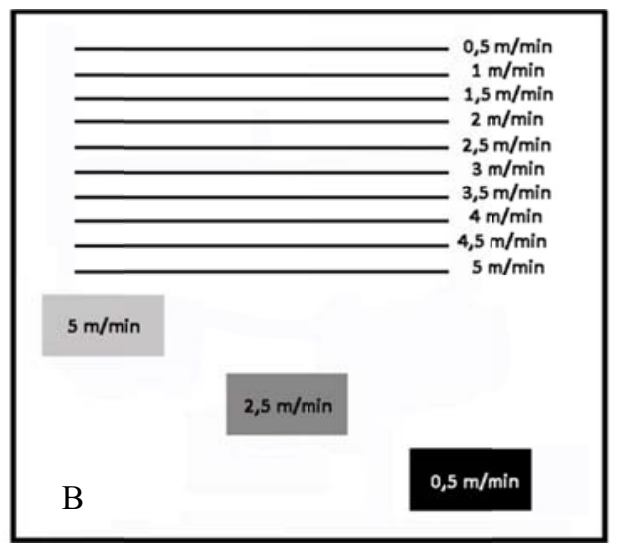

(a)

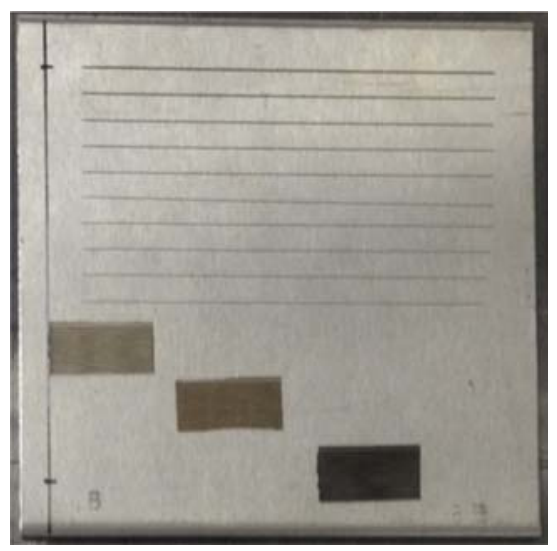

(b)

FIGURE 1. Scheme of the laser treatment realization (a) and a sample after marking process (b) 
Three identical samples were produced. After the marking process the samples were observed through a Leica DCM3D confocal microscope in order to evaluate the geometry (in particular the maximum depth and the width) and the roughness of the marks and to try to correlate the process parameters with the above mentioned features. In order to evaluate the effect of the laser marking on the Fe-Ni-Cr alloy sample surface, two of these samples were exposed to salt spray corrosion test (Angelantoni DCTC 600 P chamber), according to UNI EN ISO 9227 standard, for 480 hours. The main parameters adopted for the test were: salt solution at $5 \% \mathrm{Na} \mathrm{Cl}$ and a temperature of $50{ }^{\circ} \mathrm{C}$.

\section{RESULTS AND DISCUSSION}

In figure 2 are reported the macrographs of the marks. First of all it is possible to point out that the marks are clearly visible, ensuring the requirement of traceability for the component. It is also possible to assess that the scan speed of the laser influences both the dimensions of the marks and their superficial appearance. In fact, ranging from the lowest to the highest scan speed adopted, it is possible to observe a change of the superficial morphology of the marks. This changing is probably due to the different heat input related to the different scan speed, for the higher values of the heat input, i.e. the lower values of the scan speed, it is possible to observe a more evident oxidization of the material. In figure 3 are reported the measured features of the marks, and in particular the mark width, the maximum depth and the roughness parameters. Concerning these parameters were chosen Sa and Sz calculated following the ISO 25178 standard, such a decision because these parameters better describe the surface produced by the marking process. It is possible to observe that, as expected, the maximum depth decreases with the increasing of the scan speed, i.e. with the decreasing of the heat input. Moreover it is possible to observe a quite linear trend, this suggests that the heat input has a direct influence on the mark maximum depth. Concerning the width it is possible to observe that the width remains quite constant from the scan speed $0 \mathrm{~m} / \mathrm{min}$ to the scan speed $3 \mathrm{~m} / \mathrm{min}$, at this value it can be noticed a drop down and after that the width remains once again quite constant. This behaviour suggests that the heat input is not the main factor that rules the mark width, there are others parameters (laser beam focal spot and length, power, pulse frequency et cetera) that play an important role in the generation of the mark width.

Regarding the roughness parameters measured on the bottom of the marks, Sa and Sz, is not possible to observe a clear correlation with the scan speed. Such a result was expected because the microgeometry is ruled by the complex material flow produced during the marking by the laser beam, these material flows are also influenced by a number of factors. Nevertheless it is possible to observe a slight decreasing of the roughness values with the increasing of the scan speed, i.e. the decreasing of the heat input. An explanation of this outcome can be that with the decreasing of the heat input there is a consequent reduction of the material flow so the material to be marked retains the initial roughness, conversely with the increasing of the heat input there is an increase of the material flow that leads to an increasing of the superficial asperities and irregularities.

In figure 4 is reported a picture of a marked plate after the $480 \mathrm{~h}$-corrosion test. From a first visual inspection, no effect could be detected on the surface of the samples, this results was expected because the material under investigation has a good corrosion resistance thanks to the high Chromium content. A more detailed observation of the samples showed the presence of corrosion products on the bottom of the marks and on the surface of the sample, as reported in figure 5. These suggest the occurrence of a slight degradation of the material, but the marks remain visible and evident so it is possible to asses that this little corrosion can be neglected. 


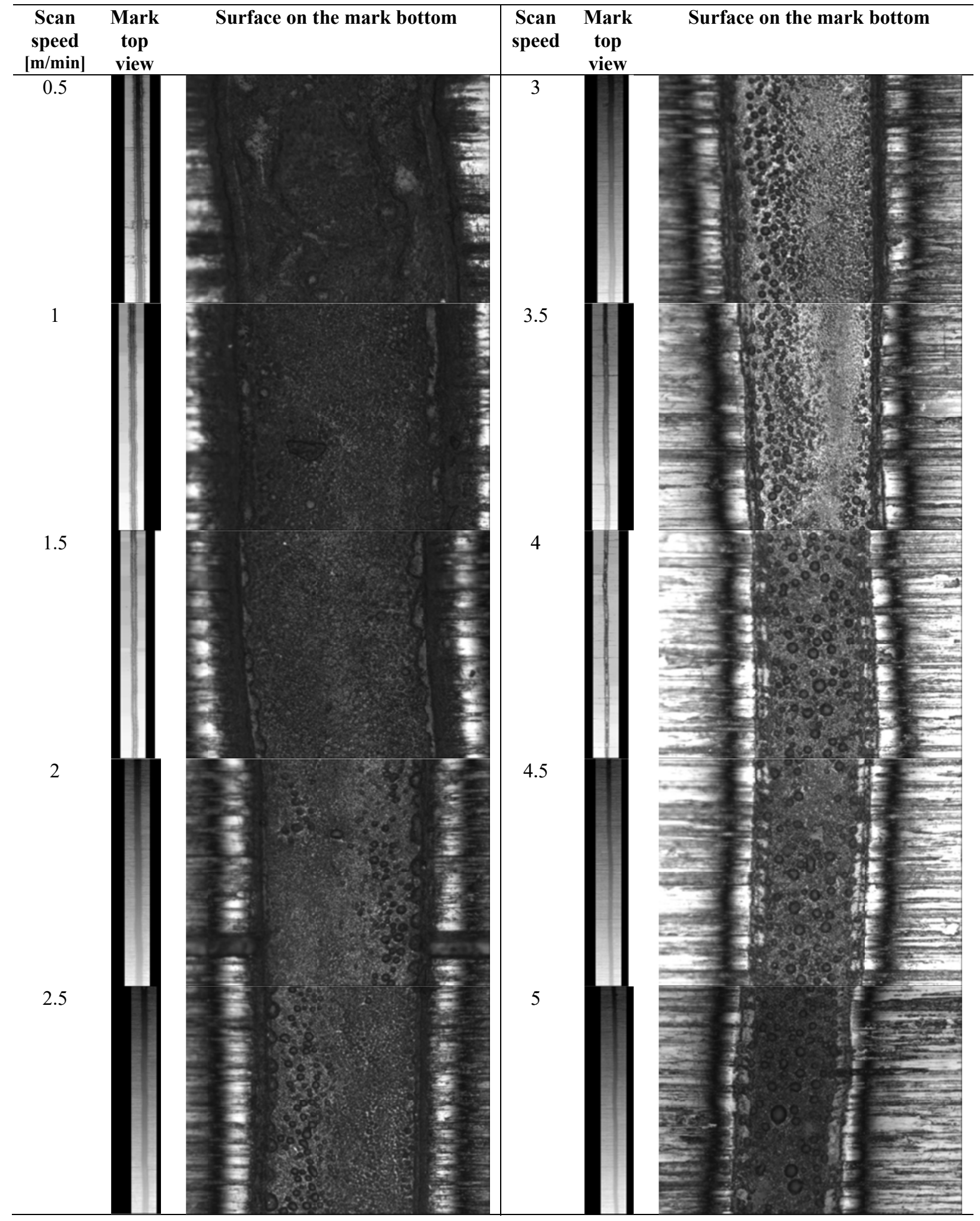

FIGURE 2. Macrographs of the top surface of the marks. 


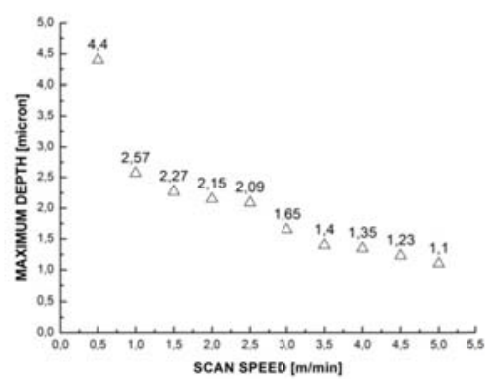

a)

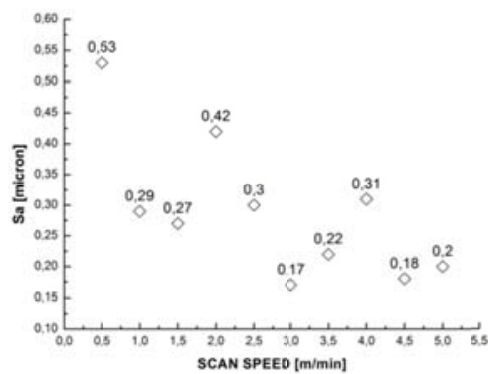

c)

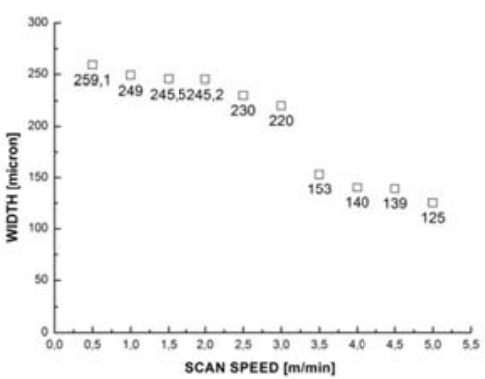

b)

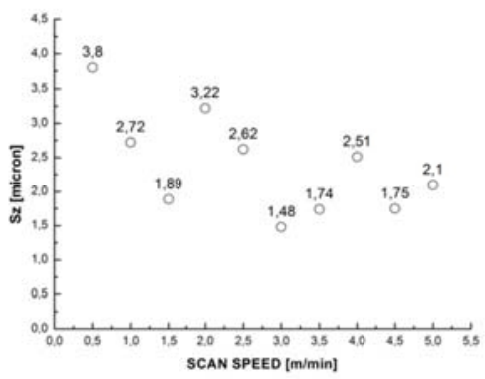

d)

FIGURE 3. Macrographs of the top surface of the marks.

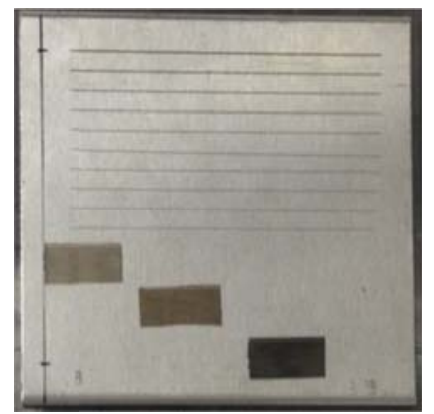

FIGURE 4. Sample after $480 \mathrm{~h}$ of salt spray chamber.

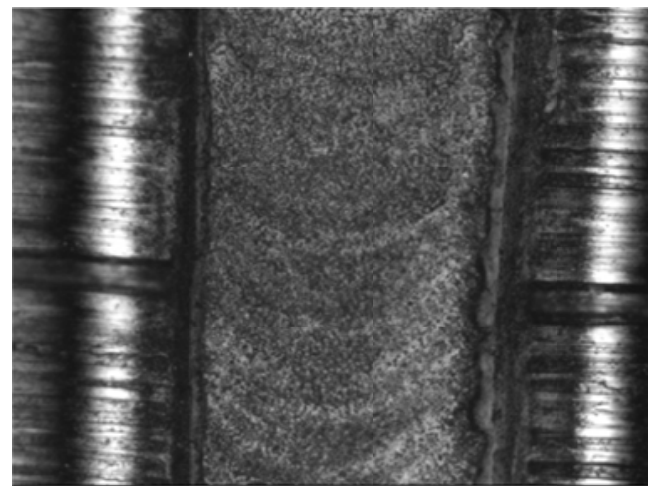

a)

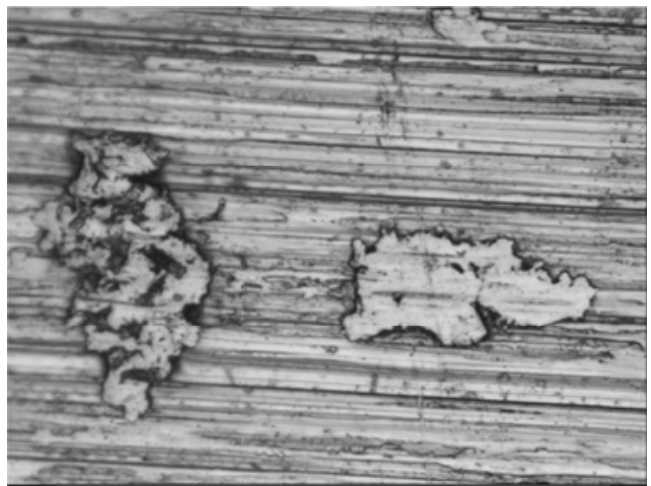

b)

FIGURE 5. Corrosion products on the bottom of the mark (a) and on the surface of the plate (b). 


\section{CONCLUSION}

Laser marking tests were performed on Fe-Ni-Cr alloy samples, adopting a $50 \mathrm{~W}$ Tm-doped fibre laser, to study the influence of the process parameters on the mark geometry and on the corrosion properties. From the results, the main conclusions are the following:

- It was proved the feasibility of the marking process by means of the adopted laser source, in fact effective marks were obtained in all the processing conditions;

- The mark geometry is influenced by the process parameters, in particular the maximum depth showed a linear correlation with the laser scan speed. Concerning the width of the marks a different and more difficult to understand trend was observed, this result suggest that the mark width is ruled by different process parameters (such as spot diameter, pulse frequency and so on) so it is more difficult to find a correlation with a single parameter;

- The roughness values measured on the bottom of the marks were quite low, moreover no evident correlation was observed between the roughness, in terms of Sa and Sz, and the laser scan speed. Such a result because the superficial roughness is ruled by complex phenomena of material flow that can not be related to only one parameter;

- The marked samples showed a good corrosion resistance, in particular no evident damages were observed after 480 hours of exposure in salt spray. This result is thank to the high chromium content of the alloy under investigation.

\section{ACKNOWLEDGMENTS}

The authors would like to acknowledge the support of Arinox, for the material supplying and IPG Photonics, Italy, which provided the laser source and the experience of its specialists.

\section{REFERENCES}

1. F.Cornacchia, A. Toncelli, M.Tonelli, 2- $\mu$ m lasers with fluoride crystals: Research and development, Progress in Quantum Electronics 33 (2009) 61-109.

2. A. Godard, Infrared (2-12 $\mu \mathrm{m})$ solid-state laser sources: a review, C. R. Physique 8 (2007) 1100-1128.

3. Hemming, N. Simakov, J. Haub, A. Carter, A review of recent progress in holmium-doped silica fibre sources, Optical Fiber Technology, in press.

4. C.W. Rudy, M.J.F. Digonnet, R.L. Byer, Advances in 2-lm Tm-doped mode-locked fiber lasers, Optical Fiber Technology, in press.

5. W. Romanowski, R. Lisiecki, H. Jelinkov, J. Sulc, Thulium-doped vanadate crystals: Growth, spectroscopy and laser performance, Progress in Quantum Electronics 35 (2011) 109-157.

6. C. Mandolfino, E. Lertora, S. Genna, C. Leone, C. Gambaro, Effect of laser and plasma surface cleaning on mechanical properties of adhesive bonded joints, Procedia CIRP 33 (2015) 458-463, http://dx.doi.org/10.1016/j. procir.2015.06.054.

7. Astarita, Genna, Leone, Memola Capece Minutolo, Squillace, Velotti - Study of the laser marking process of cold sprayed titanium coatings on aluminum substrates - Optics \&Laser Technology 83 (2016) 168-176. http://dx.doi.org/10.1016/j.optlastec.2016.04.007. 